\title{
Preliminary Study of Pressure Wave High Lift Aided Device and Basic Modeling of Its Efficiency
}

\author{
Xinyan $\mathrm{Fu}^{1}$ and Ganlin $\mathrm{Lv}^{*, 2}$ \\ ${ }^{1}$ Huazhong Agriculture University, Hubei, 430070, P.R. China \\ ${ }^{2}$ Beihang University, Beijing, 100191, P.R. China
}

\begin{abstract}
In recent disaster relief operations, short take-off and landing aircrafts began to excel. To enable the large fixed wing aircrafts to take off in a shorter distance, we designed a new high lift aided device. The design principle is different from conventional ones. According to formula of lift, lift is proportional to air density. Pressure wave high lift aided device is the lift-promoting system functioning by using pressure wave to increase the air density around wings. This article shows the mechanic design and the lift-promoting efficiency calculation. The calculation is based on a simplified model of Boeing 747-400. Since the take-off process is a variable acceleration motion, it is difficult to confirm the distance an aircraft need to reach take-off speed. We turned to study the extra take-off weight at the original take-off speed. We found that with the help of this device, the maximum take-off weight has increased $2.62 \%$. And the take-off weight increment is proportional to the amplitude and frequency of the device. The author's conclusion is that although the increase is not much, the benefit is obvious. It could be more efficient if the amplitude and frequency are enhanced.
\end{abstract}

Keywords: Wave high lift, aided device, take-off, mechanic design.

\section{INTRODUCTION}

Civil aircrafts are required to have good take-off and landing performance to ensure their overall performance. For a typical large twin-engine civil aircraft [1]:

1) If the lift coefficient is improved 0.1 , equivalently to $1^{\circ}$ decrease in approach attitude angle, at the given landing attitude angle, the landing gear can be shortened to save $635 \mathrm{~kg}$ weight.

2) At the same approach speed, a $1.5 \%$ increase in maximums lift coefficient means $3000 \mathrm{~kg}$ more load.

3 ) In the take-off state, $1 \%$ increase in lift-drag ratio can lead to $1270 \mathrm{~kg}$ more loads or $280 \mathrm{~km}$ more voyage.

We can notice that the overall performance of the plane is sensitive to the efficiency of high lift device. Now, most high lift devices on the plane are the flaps rising devices, including leading edge slats rising device, trailing edge raising device, leading edge flap and their combination. With long time development of high lift system, it has matured in many aspects. However, the process of development has not finished. The performance of high lift system and lift-drag ratio still could be enhanced [1].

The main design principle in conventional high lift devices is increasing $C y$ through flaps or slats and

*Address correspondence to this author at the School of Advanced Engineering, Beihang University, Beijing, 100191, P.R. China;

E-mail: Ganlin_lv@buaa.edu.cn re-accelerating the air fluid to put off boundary layer separation [2]. There has not been a design to enhance the raising performance functioning by changing partial air character.

In this study, we try to use pressure wave to compress the air partially, which could create a denser flow field, so that the aircrafts will be able to take off and land in a shorter distance $[2,3]$.

\section{FORMULATION}

\section{Principle of Pressure Wave High Lift Aided Device}

According to general formula:

$F_{y}=(1 / 2) \rho S C_{\gamma} v_{a}^{2}$

Where lift $F_{y}$ is in direct proportion to the air density $\rho$ when other parameters remain the same. The main principle of Pressure Wave High Lift Aided Device (PWHLAD) is to enhance air density around winds through pressure wave. As a result, the aircrafts could obtain extra lift [4].

Also, we know that the formula of drag is:

$F_{x}=(1 / 2) \rho S C_{x} v_{a}{ }^{2}$

As lift-drag ratio is approximately the ratio of lift of wings and drag of the whole aircraft, the overall lift-drag ratio will increase $\rho^{\prime} / \rho$ when the air density around wings has an increase of $\rho$ '. This means that the lift have a larger proportion of increase than the drag so that the take-off and landing performance could be optimized [5]. 


\section{Increase of Air Density}

The movement of pressure wave is non-linear. But if we exert the restriction that the amplitude is much less than the wavelength, approximately, we can study the process in linear acoustic area.

Focusing on an infinitesimal air mass, according to Law of Conservation of Mass, we can elicit this formula [2, 3]:

$\frac{\partial \rho}{\partial t}=\frac{\partial(\rho v)}{\partial x}$

This formula indicates that the increase in air density in unit time is equal to the air mass flow in unit distance. If we study small amplitude sound wave (usually that is the case) and neglect the high order small, the formula above could be rewrite as [3]:

$\frac{\partial \rho^{\prime}}{\partial t}=-\rho_{0} \frac{\partial v}{\partial x}$

Then we study the sound wave as a sine wave. We would get the change of air density [4]:

$\rho^{\prime}=\rho-\rho_{0}=\left(A \rho_{0} \omega / c\right) \cos \omega(t-x / c)$

\section{SPECIFIC DESIGN SCHEME OF PRESSURE WAVE HIGH LIFT AIDED DEVICES}

PWHLAD should be installed bilateral symmetrically in specialized cabins inside the fuselage. The specialized cabins are located in the near front of wing roots and the cabins need to be at the same level as the wing roots. When an aircraft is taking off, the cabins would be opened and the PWHLAD will begin to work if the aircraft reaches the proper speed. The pressure wave will go along the wings (Fig. 1). After taking off, the PWHLAD will stop working and the cabins will be closed.

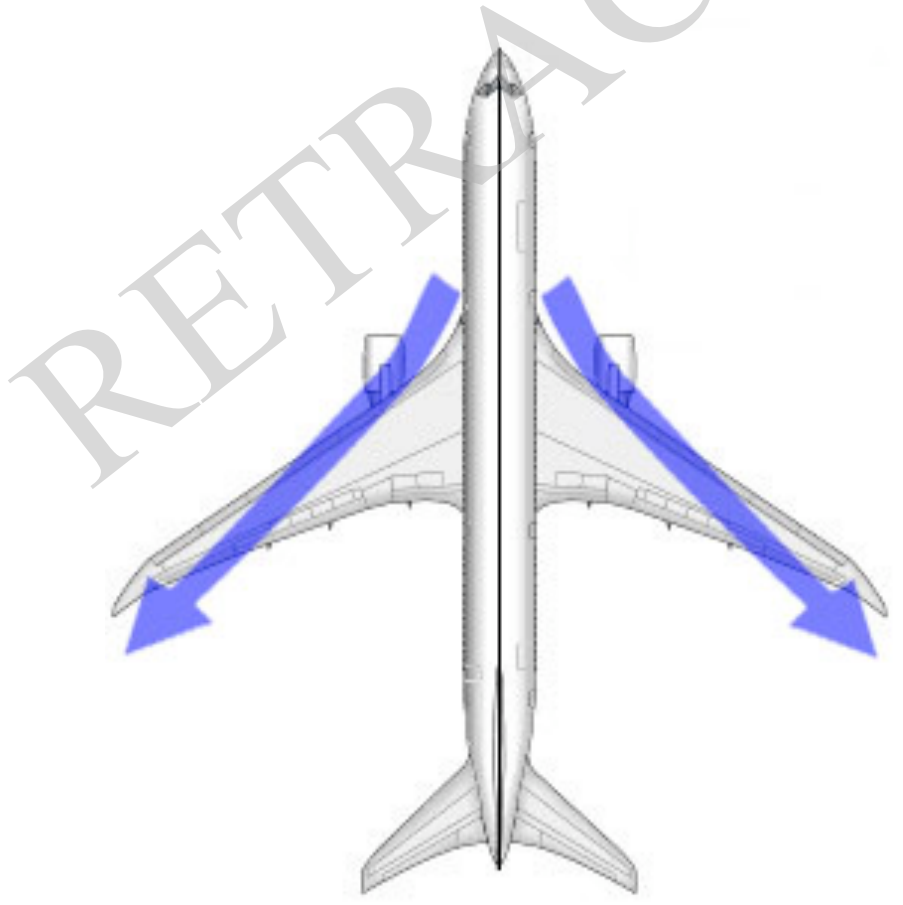

Fig. (1). Location of the specialized cabins and PWHLAD.
The PWHLD mainly contains five parts, as shown in the (Fig. 2). We number the core parts: The white circle is $\mathrm{A}$, the black is $\mathrm{B}$, the blue is $\mathrm{C}$ and the red line is $\mathrm{D}$. The yellow belts are $\mathrm{E}$ :

1. A and B represent two pressure wave generator

2. C represents a mechanical wave filter

3. D represents spindle

The two generators are at opposite phases (shown by the color of black and the color of white). The mechanical filter is installed on the spindle and the mechanical wave filters on both sides share one spindle. They rotate around spindle driven by a high speed electrical motor. The pressure wave generators and the electrical motor have the same frequency [6].

The mechanical filter is designed to cut off the pressure wave periodically. When one generator is separated with outside atmosphere, another air inlet (not presented in the Fig. 2) will be open to re-pressurize the air around the generator, preparing for next generation. E means the allowed motion range which is two times the amplitude of generator.

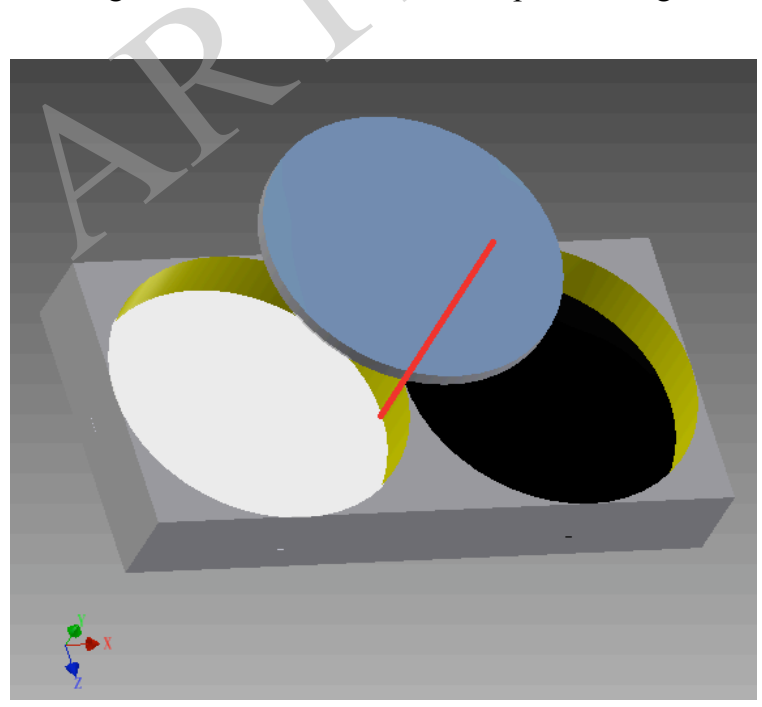

Fig. (2). The core part of PWHLAD on the right side.

\section{EFFICIENCY CALCULATION}

\section{Efficiency of Mechanical Wave Filter}

The efficiency calculation is based on the following model:

1) When the frequency of the generator is not very high, the air could be regarded as perfect gas [5].

2) Edge effect is neglected.

3) As the amplitude is small, in the calculation of $\eta$, which is the derivative of mass flux (total mass of air traveling through the generator's surface in unit time), the motion range of generator is approximately zero.

Take a pair of generators on one side as an example:

$\eta=S v \rho$ 
Where $S$ is the area and $v$ is the equation of motion of pressure wave generator, $\rho$ is the equation of air density. The derivative of mass flux without being blocked by the mechanical wave filter is:

$$
\eta_{0}=A \omega \rho_{0} \pi R^{2} \sin (\omega t)[1+(A \omega / c) \cos (\omega t)]
$$

When the pressure wave travel through the filter, it is weaken. But if we get the correct phase of the filter, the mass flux would become positive. That means the PWHLAD could enhance the air pressure around it. After the wave traveling through the air periodically, the whole wing area will get an increase in air density.

By integrating the derivative of mass flux in nonoverlap area in one period, we got of mass flux blocked by the filter is:

$$
\begin{aligned}
\Phi^{1}= & A \rho_{0} R^{2}[2(\pi-\varphi)+\sin (\varphi)-\pi \cos (\varphi)+\varphi \cos (\varphi)] \\
& -\left(A^{2} \omega \rho_{0} R^{2} / 12 c\right)[8 \sin (\varphi)-7 \sin (2 \varphi)+6(\pi+\varphi)]
\end{aligned}
$$

To make the system effective, the amplitude and angular frequency should be large enough. In the reasonable region, we choose amplitude of $2 \mathrm{~cm}$ and frequency of $167 \mathrm{~Hz}$ as a sample. We assume that the altitude of airport is at the sea level, so the sound speed is $340 \mathrm{~m} / \mathrm{s}$.

$\Phi^{1}$ has the maximum: $\Phi_{\text {max }}^{1}=0.04 \mathrm{~A} \omega \rho_{0} \mathrm{R}^{2}$.

We got the maximum positive mass flux without mechanical wave filter is: $\Phi_{\max }^{0}=0.06 \mathrm{~A} \omega \rho_{0} \mathrm{R}^{2}$.

As a result, the efficiency of mechanical wave filter is $66.67 \%$.

\section{Overall High Lift Efficiency} low:

The calculation is based on the model and assumption be-

1. We only study one side of the aircraft.

2. The model plane is as same size as Boeing 747-400, whose wing span is $60 \mathrm{~m}$.

3. To simplify calculation, we assume that the sweepback angle is $0^{\circ}$ while the wing has the same width everywhere.

4. The overall weight is $G$.

5. The take-off speed is $300 \mathrm{~km} / \mathrm{h}$.

6. Set a one-dimensional coordinate along the front edge of wing. Choose the intersection point of the front edge and fuselage as origin point.

7. The damped wave equation in the air is:

$p=p_{0} \exp (-\alpha x)$

Where $\alpha$ is the decay coefficient, $\mathrm{x}$ is the coordinate based on assumption (6). The empirical value of $\alpha$ is $1.6 \mathrm{x}$ $10^{-7} f^{2} . f$ is the vibration frequency [4].

8. Regard the air as perfect gas.

9. Without the aid of PWHLAD, at the moment that the aircraft leaves the ground, the gravity is balanced with lift. So we have:
$G=F_{y}=(1 / 2) \rho_{0} v^{2} C_{y} S$

10. Use the same parameters above that amplitude is 2 $\mathrm{cm}$, the frequency is $167 \mathrm{~Hz}$, the sound speed is 340 $\mathrm{m} / \mathrm{s}$.

The radius of generator is $1 \mathrm{~m}$.

Because of the parallel connection of two generators and the radius of generator is great smaller than the length of a wing, two filtered pressure waves can be regarded as two relatively complemented waves. As a result of this, we can approximately regard the new wave shape in $\mathrm{x}$ axis is periodically present of two filtered sine wave. The wavelength of this new wave is half that of original sine wave. The new wave is decaying as it travels complying with assumption (5).

According to the principle of relativity, the air flows at a speed of $340 \mathrm{~m} / \mathrm{s}$ through the wings when the model aircraft takes off. In order to cover the wings with compound pressure wave, the direction of original wave should have an angle of $13.77^{\circ}$ with $\mathrm{x}$ axis.

We will derive the new function of increase in air density in consideration of amplitude decadence without the effect of mechanical wave filter:

$$
\rho^{\prime}=\left(A \omega \rho_{0} / c\right) \exp (-\alpha x) \cos [\omega(t-x / c)]
$$

Eliminate the time factor $t$ by integrate $\rho$ ' in half period at a proper beginning phase and then we can get the average increase in air density:

$$
\Delta \rho=\rho_{0}(2 A \omega \beta / \pi c) \exp (-\alpha x)
$$

Finally, after another integral in regard of $\mathrm{x}$ and use the assumption (7), we have the overall formula of lift:

$$
F=\left[1+\frac{2 A \omega \beta}{\pi c \alpha L}\left(1-e^{-\alpha L}\right)\right] G
$$

In this model, $F=(1+2.62 \%) G$

\section{Efficiency Analysis}

1. In the model above, the wavelength is $2 \mathrm{~m}$ and the amplitude is $2 \mathrm{~cm}$. It is corresponding with the premise that the amplitude is much less than the wavelength. So the linear hypothesis of wave is rational [6].

2. In this model we assume that the sweepback angle is $0^{\circ}$, which is not corresponding with $30^{\circ}$ sweepback angle used in current large aircraft. According to the load distribution in reality, the equivalent point of lift is closer to the wing butt. That means the pressure wave will has less decadence and the efficiency will increase.

3. We considered absorption attenuation and scattering attenuation. But the diffusion attenuation is neglected. This simplification will make calculated value a little larger than actual value. 


\section{CONCLUSION}

PWHLAD can enhance the lift when the aircraft is taking off and landing. According to the efficiency calculation based on a simplified large aircraft, PWHLAD could provide an increase of $2.62 \%$ in total lift. For 300-400 tons large aircrafts, the lift could increase 7.86-10.4 tons at the original weather condition and take-off speed. As the increase in lift is proportional to the weight of the aircraft itself, the increase will become larger as the plane is heavier. It will allow An225 to carry 15.7 tones more load. Load capacity is one of the most important indicators to an aircraft. PWHLAD is benefit to promote the overall performance, especially the taking-off performance and load capacity.

\section{NOMENCLATURE}

\begin{tabular}{|c|c|c|}
\hline 4 & $=$ & amplitude of oscillatic \\
\hline$\alpha$ & $=$ & the decay coefficient \\
\hline$\beta$ & $=$ & efficiency of mechani \\
\hline$c$ & $=$ & velocity of sound at \\
\hline$C x$ & $=$ & force coefficient in tl \\
\hline$C y$ & $=$ & force coefficient in $t$ \\
\hline$d t$ & $=$ & time step \\
\hline$F x$ & $=$ & $\begin{array}{l}X \text { component of the } \\
\text { acting on the vehicle }\end{array}$ \\
\hline$F y$ & $=$ & $\begin{array}{l}Y \text { component of the } \\
\text { acting on the vehicle }\end{array}$ \\
\hline$f$ & $=$ & vibration frequency \\
\hline$h$ & $=$ & height \\
\hline$\rho$ & $=$ & air density \\
\hline
\end{tabular}

$\rho_{0} \quad=$

$\rho^{\prime} \quad=$

$\Delta \rho \quad=$

$\Phi=$

$\eta \quad=$

derivative of mass flux (total mass of air traveling through the generator's surface in unit time)

$R=$ the radius of pressure wave generator

$v_{a}=$ aircraft velocity

$v \quad=\quad$ flow velocity

$\omega=$ angular frequency

\section{CONFLICT OF INTEREST}

The authors confirm that this article content has no conflict of interest.

\section{ACKNOWLEDGEMENT}

Declared none.

\section{REFERENCES}

[1] Z. Ziqiang, and Y. Chen, "Aerodynamic design OF modern aircraft's," National Defense Industry Press, Beijing, 2011.

[2] D. A. John, "Fundamentals of Aerodynamics," McGraw-Hill, New York Press, 1, 2, 2010.

[3] Zuwen Qian, “Non-linear Acoustics," Science Press, Beijing, 1992.

[4] D. Lin, "Foundation of Recording Acoustics," China Broadcast Press, Beijing, pp. 203-212, 2003.

[5] R. Feng, "Ultrasound Manual," Nanjing University Press, Nanjing, pp. 26-28, 1999.

[6] S. Feng, "Introduction of Ocean Science," Advanced Education Press, Beijing, 1999.

(C) Fu and Lv; Licensee Bentham Open.

This is an open access article licensed under the terms of the (https:/creativecommons.org/licenses/by/4.0/legalcode), which permits unrestricted, noncommercial use, distribution and reproduction in any medium, provided the work is properly cited. 